\title{
Comportamiento juvenil universitario en busca de la felicidad: su caracterización y su eficacia*
}

\section{Youth Behavior in College to Search of Happiness: Characterization and Effectiveness}

Recibido: febrero 22 de 2013 | Revisado: febrero 8 de 2014 | Aceptado: junio 21 de 2014

\author{
MAURICIO TORRES DeIK ** \\ EMILIO MOYANO-DÍAZ*** \\ Universidad de Talca, Chile \\ DARÍO PÁEZ ***** \\ Universidad del País Vasco, España
}

doi:10.11144/Javeriana.UPSY13-4.cjub

Para citar este artículo: Torres, M., Moyano-Díaz, E., \& Páez, D. (2014). Comportamiento juvenil universitario en busca de la felicidad: su caracterización y su eficacia. Universitas Psychologica, 13(4), 1419-1428. http://dx.doi.org/10.11144/Javeriana. UPSY13-4.cjub

* Grupo de Investigación en Calidad de Vida y Ambientes Saludables, Facultad de Psicología, Universidad de Talca, Chile. Correspondencia respecto del artículo a: emoyano@utalca.cl

*** Facultad de Psicología. Correo electrónico: mtorresdeik@gmail.com

**** Profesor Titular. Coordinador Área de Psicología Social y de las Organizaciones. Facultad de Psicología. Coord. Grupo de Investigación en Calidad de Vida y Ambientes Saludables (GICVAS). Correo electrónico: emoyano@utalca.cl

***** Profesor Catedrático, Facultad de Psicología, Dpto. Psicología Social y Metodología de las Ciencias del Comportamiento.Correo electrónico: dario.paez@ ehu.es

\section{RESUMEN}

Se busca determinar qué comportamientos realizan los jóvenes para procurarse estados de felicidad y su eficacia en ello. Participan 433 estudiantes universitarios respondiendo un cuestionario (CCPF), para medir la frecuencia y eficacia de las conductas emitidas para procurarse felicidad, y la Escala de Felicidad Subjetiva de Lyubomirsky y Lepper, controlándose las variables sexo, religión y personalidad autoadscrita. Se observan niveles moderados de felicidad, sin registrarse diferencias según sexo, religión o personalidad autoadscrita. Los jóvenes desarrollan frecuentemente conductas para aumentar la felicidad -las mujeres más que los hombres-que resultan moderadamente eficaces. Las mujeres realizan más actividades de afiliación, religiosas y de ocio pasivo, y los hombres, más físicas y de metas instrumentales. La frecuencia y la eficacia de las conductas realizadas para aumentar la felicidad predicen de forma significativa y positiva las puntuaciones de felicidad. Los puntajes más altos de felicidad corresponden a quienes se autoclasifican como extrovertidos.

Palabras clave

conductas pro felicidad; jóvenes; estrategias conductuales

\section{A B S T R A C T}

It seeks to determine what behaviors are performed to get happiness and his efficiency. We evaluated 433 college students who completed a questionnaire (CCPF) constructed to measure the frequency and efficiency of conduct issued to increase happiness, and Subjective Happiness Scale of Lyubomirsky and Lepper, controlling the variables sex, religion and self-ascribed personality. In general, there is moderate levels of happiness without registering differences in this according to sex, religion or self-ascribed personality. The students frequently perform behaviors to increase happiness -women more than men - and are reported as moderately effective. Women perform more activities of affiliation, religious and passive leisure, and men more physical activities and related to instrumental goals. The frequency and effectiveness of actions carried out to increase the happiness predict significant and positively happiness scores. Higher happiness scores correspond to those who classify themselves as extroverts.

Keywords

Pro-happiness behaviors; youth; behavioral strategies 


\section{Introducción}

Un motivo humano central es la búsqueda de la felicidad y las personas se esfuerzan por conseguirla utilizando diferentes estrategias o conductas (Gratz, 2003), aprendiendo cuáles de estas aumentan aquella. Sin embargo, hay comportamientos que causando un efecto emocional placentero inmediato tales como plantearse objetivos no realistas, drogarse legal o ilegalmente $\mathrm{u}$ otros, pueden traer consecuencias negativas a mediano o largo plazo en la salud física y el bienestar psicológico (Huesmann, Moise-Titus, Podolski, \& Eron, 2003; Pahl, Brook, Koppel, \& Yeon, 2011).

Entre las actividades realizadas para procurarse felicidad están compartir con amigos y practicar actividades artísticas y físicas (Hsiao \& Thayer, 1998; Michalos, 2005; Schnittker, 2008), proponerse alcanzar o lograr metas trascendentes (Brunstein, 1993). Concordante con esto, las investigaciones centradas en la regulación emocional han proporcionado evidencia de una relación de causalidad entre la conducta intencional y los cambios de ánimo. Hsiao y Thayer (1998) reportaron que ejercitarse físicamente es un buen regulador del ánimo y de las emociones positivas, y quienes practican algún deporte tienen mayores niveles de felicidad que quienes no lo hacen. En dirección convergente Pavez, Mena y Vera (2011) han mostrado el rol protector de la felicidad respecto de la ansiedad estado y rasgo.

Un análisis de la literatura muestra que los comportamientos para conseguir felicidad son clasificables en distintos ámbitos de vida: social y de consumo, espiritual (religiosidad y artístico, principalmente), de metas personales y físico deportivo. A continuación, se resume alguna evidencia acerca de ello.

Lyubomirsky, Sheldon y Schkade (2005) comprobaron que el apoyo social suele aumentar los estados de ánimos positivos. Para la mayoría de la gente, el apoyo familiar y de los amigos aumenta la felicidad; este último puede tener efectos mayores que el apoyo del cónyuge (Schnittker, 2008). También, ha sido señalado que las mujeres utilizan más frecuentemente el apoyo social para disminuir los estados de ánimo negativos (Melin, Fugl-Meyer, \&
Fugl-Meyer 2003). Por su parte, Diener y Seligman (2002) mostraron en 200 estudiantes universitarios que los más felices dedicaban mucho de su tiempo a relacionarse con amigos, cultivando relaciones constructivas.

En la actividad social es frecuente el consumo de alcohol, y en el caso de los adolescentes ello ha sido relacionado con la aceptación de los pares y un mejor ajuste psicosocial (Newcomb \& Bentler, 1988), con un aumento del ánimo positivo, un incremento de la confianza en sí mismo y un mejor ajuste social (Orford, Krishnan, Balaam, Everitt, \& Van der Graaf, 2004). Sin embargo, quienes consumían más alcohol tenían menor rendimiento en sus trabajos y menor satisfacción con su salud, siendo más propensos a padecer malestar, ansiedad y depresión (Casswell, You Ru Quan, \& Huckle Taisia, 2011). Comer para procurarse estados placenteros e ingerir alimentos ricos en azúcar puede causar un efecto positivo sobre la felicidad, similar al experimentado en las adicciones (Parylak, Koob, \& Zorrilla, 2011). Sin embargo, comer compulsiva o excesivamente es un comportamiento que realizado junto con prácticas de ocio como ver televisión, resulta poco saludable por el sedentarismo, riesgo de sobrepeso y obesidad asociados (Soto y Moyano-Díaz, 2010), con efectos negativos sobre la salud y la felicidad.

Otras conductas con efectos agradables en lo inmediato y daño a largo plazo es el consumo de tabaco y drogas. Consumir nicotina durante un tiempo prolongado produce síntomas de ansiedad, y cuando su ingesta disminuye o termina abruptamente podría provocar depresión. Quienes consumen mucho tabaco reportan un deteriorado bienestar psicológico y baja satisfacción con la vida (Pahl, Brook, Koppel, \& Yeon, 2011). A la vez, la marihuana es una de las drogas ilegales más consumida por los jóvenes (Mcleod et al., 2004) y durante los estudios universitarios aumenta su consumo junto con el del tabaco y alcohol y, así, el riesgo de enfermedades relacionadas al sedentarismo (sobrepeso), perjudicando el bienestar (Brandao, Pimentel, \& Cardoso, 2011)

Entre los aspectos psicológico-espirituales relativos a la felicidad se ha observado que quienes practican la gratitud -entendida como una 
emoción de empatía, una actitud o una respuesta de afrontamiento dirigida a otras personas, a la naturaleza o a seres no humanos, entre otros (Teigen, 1997) - son más propensos a la felicidad, la vitalidad, el optimismo y esperanza, así como a una mayor satisfacción con la vida (Lazarus \& Lazarus, 1994). Gallup (1998) reportó en adolecentes y adultos estadounidenses que más del 90 \% señaló que expresar agradecimiento les ayudó a sentirse muy felices o un poco más felices. McCullough, Emmons y Tsang (2002) argumentan que expresar gratitud disminuye las probabilidades de experimentar emociones negativas como la ansiedad, la depresión y la envidia. Vinculado a esto, algunos estudios han reportado una relación de baja magnitud entre religiosidad y felicidad (Diener \& Seligman, 2002; Pollner, 1989) aunque ello no parece ser universal, o está matizado por otras características societales, ya que por ejemplo, lituaneses y eslovacos religiosos o creyentes reportaron bajos niveles de felicidad (Kesebir \& Diener, 2009).

La práctica del arte aparentemente promueve la felicidad; Michalos (2005) reportó una correlación positiva entre la realización de actividades artísticas como cantar, tocar instrumentos musicales, entre otras, y la felicidad, independientemente de la frecuencia de su práctica. Laukka (2007) señala que escuchar música es una actividad de ocio muy frecuente y que regula los estados de ánimo, provoca placer y relaja a los oyentes, generando recuerdos emotivos que incrementan las emociones positivas. Otra estrategia para aumentar emociones positivas es ejercer control mental dirigiendo la atención hacia los pensamientos o actividades realizadas en el aquí y ahora, a circunstancias positivas, a cosas que podrían ser aún mejores, evitando focalizarse en situaciones negativas de la vida (Erisman \& Roemer 2010). Larsen y McKibban (2008) encontraron que enfocar el pensamiento a situaciones negativas correlaciona negativamente con la felicidad, y ha sido sugerido mejorar los estados de ánimo, comunicándose y celebrando eventos positivos con los demás (Langston, 1994).

En el ámbito de las metas personales, Brunstein (1993) reportó en estudiantes universitarios que el compromiso con sus objetivos personales, la posibilidad de alcanzarlos y el progreso hacia su consecución correlacionaban con la felicidad. Sin embargo, otras investigaciones han mostrado efectos que parecen paradójicos, por cuanto personas que realizan actividades convencidas que estas les provocarán felicidad, obtienen, por el contrario, disminución de sus estados de ánimos positivos (Gratz, 2003). Es así como Gui y Stanca (2009) han observado que personas que tienen bajo autocontrol y que miran televisión más de 2.5 horas por día reportan puntuaciones bajas de felicidad.

\section{Medición de conductas para provocarse felicidad}

Aunque existen instrumentos para medir felicidad, por ejemplo, Alarcón (2006) o Moyano-Díaz, Flores y Soromaa, (2009) entre otros, son extremadamente escasos en cambio los destinados a identificar comportamientos, actividades o estrategias personales para procurarse felicidad. Una excepción es el cuestionario Happiness Inventory Scale (HIS) de Takch y Lyubomirsky (2006) cuya construcción comenzó con una encuesta abierta a 70 estudiantes universitarios para que anotaran las actividades que realizaban para aumentar su felicidad. De allí se desarrolló un instrumento de 55 ítems agrupados en 8 dimensiones o estrategias para procurarse estados de felicidad, a saber: afiliación social, fiestas y clubes, control mental, búsqueda de objetivos instrumentales, ocio pasivo, ocio activo, religión, intentos directos. Los autores señalan que las actividades o comportamientos más relacionados con altos niveles de felicidad corresponden a las dimensiones afiliación social (ayudar a otros, comunicarse con los amigos), religiosidad, ocio activo (practicar deporte), la obtención de metas instrumentales (organizar la vida) y las conductas emocionales positivas (actuar feliz, sonreír, etc.). Por otra parte, conductas relacionadas con el ocio pasivo -ver televisión, dormir, etc.- y el consumo de alcohol y drogas correlacionan negativamente con la felicidad (Takch \& Lyubomirsky, 2006). A pesar que existen reportes y algunos instrumentos para evaluar la relación entre conducta y felicidad en países anglosajones (Lyubomirsky, Sheldon, \& 
Schkade, 2005; Schimmack, Schupp, \& Wagner, 2008; Takch \& Lyubomirsky, 2006; Warner \& Vroman, 2011), se desconoce si estas prácticas conductuales son generalizables a distintas culturas y, si lo fueran, si alcanzan un nivel de efectividad semejante a la de la cultura de origen.

Como sabemos, la felicidad depende en una alta proporción de aspectos que las personas son capaces de modificar en su vida cotidiana (Lyubomirsky et al., 2005) y en el presente estudio se busca identificar qué conductas despliegan los jóvenes universitarios para procurarse felicidad. Se espera identificar la relación entre estas conductas o tipo de actividades y su efecto eventual en la felicidad, evaluando su eficacia y, por último, determinar cómo se relacionan con el género, la adscripción religiosa y el rasgo de personalidad (auto-adscrito) extraversión o introversión, variables que han sido identificadas como relevantes al respecto en la literatura científica. Se espera encontrar diferencias según género: mujeres más felices que los hombres, hombres y mujeres con comportamientos o estrategias modales distintas, hombres realizando más frecuentemente que las mujeres ejercicio físico y éstas realizando una mayor cantidad de conductas relativas a apoyo social para provocarse estados de ánimo positivos. También, que los más felices practiquen alguna religión o tengan alguna creencia religiosa en contraste con quienes no, que tengan una mayor actividad de interacción con otras personas, que realicen conductas destinadas a celebrar y, también, que se autoclasifiquen en el rasgo extroversión.

\section{Método}

Es un diseño no experimental, transversal, para describir y relacionar los comportamientos y estrategias desplegadas por los participantes para aumentar su felicidad. Son 433 estudiantes universitarios, 167 (38.6\%) hombres y 266 (61.4\%) mujeres, entre 18 y 31 años $(M=22.12 ; D E=2.48)$ de los cuales un $61 \%$ pertenece o practica alguna religión.

\section{Instrumentos}

Se utiliza la Escala de Felicidad Subjetiva (EFS) de Lyubomirsky y Lepper (1999) y un cuestionario que se denominó Cuestionario de Comportamientos Pro Felicidad (CCPF) elaborado a partir del HIS, más algunos ítems para registrar sexo, religión y personalidad auto-adscrita. La EFS tiene 4 ítems de formato de respuesta Likert de 7 niveles, donde a mayor puntaje mayor felicidad. Se utiliza la versión chilena (Moyano y Ramos, 2007), de adecuada consistencia interna (alfa de Cronbach de 0.79 y de 0.77 para test-retest de 10 semanas en 133 estudiantes universitarios) de estructura monofactorial, con $61.08 \%$ de varianza explicada y correlación de 0.67 con la Escala de Satisfacción Vital de Diener (validez convergente). Además, la EFS muestra validez convergente con escalas de inteligencia emocional, bienestar psicológico y regulación afectiva adaptativa en muestras de México, España y Chile (Páez, Mendiburo-Seguel, Martínez-Sanchez, \& Sevillano, 2013). El cuestionario CCPF se usa para identificar qué conductas utilizan estudiantes universitarios para procurarse felicidad teniendo como base el HIS de 55 ítems de Takch y Lyubomirsky (2006) del que se mantuvieron sus 8 dimensiones y una mayoría -aunque no todos- de sus ítems, ya que algunos no resultaban adecuados al contexto cultural chileno, por lo que se adaptaron a él o se eliminaron, por ser infrecuentes o alejados de las normas culturales locales. Además, se le cambió el formato de respuesta para que fuera más fácil de contestar, usando un recorrido menor. El CCPF quedó conformado por 44 ítems, con un formato de respuesta Likert (tanto para la frecuencia de la conducta como para su eficacia) reducido a 4 niveles donde, para frecuencia: $1=$ Nunca o casi nunca y 4 = La mayor parte del tiempo, o todo el tiempo y para efectividad: 1 = Nunca o casi nunca es efectiva y 4 = Bastante efectiva, en reemplazo de los siete niveles propios del HIS para ambas dimensiones. Las ocho dimensiones del CCPF al igual que el HIS de Tkach y Lyubomirsky (2006) son: afiliación social, fiestas y discotecas, control mental, búsqueda de objetivos instrumentales, ocio pasivo, ocio activo, religión, intentos directos. El CCPF presenta buena confiabilidad global (alfa de Cronbach: 0.9) y adecuada para ambas secciones del instrumento (para frecuencias $\alpha=0.78$ y para eficacia $\alpha=0.88$ ), así como para sus 8 dimensiones (fluctuantes entre 0.69 y 0.81). 
Respecto de personalidad, el respondiente debía leer las definiciones de los rasgos extraversión-introversión ofrecidas y autoclasificarse en una de ellas, en sustitución de un instrumento como el NEOFI para evitar un instrumento extenso.

\section{Procedimiento}

El CCPF fue construido mediante un procedimiento similar al utilizado para el HIS, solicitando a 50 estudiantes universitarios de distintas carreras, elegidos por conveniencia o disponibilidad, que anotaran cuáles eran las conductas que realizaban para aumentar su felicidad y la eficacia de cada una. Se identificaron así 200 conductas, que mayoritariamente coincidieron con las incluidas en el HIS de Takch y Lyubomirsky (2006), aunque hubo algunas pocas que resultaron nuevas, tales como: "compartir con la familia" y "pololear o andar"'. Las actividades mencionadas coinciden con estudios de muestras chilenas, en las que compartir con amigos y familiares, iniciar relaciones amorosas y ser aceptados en grupos sociales valorados, son mencionadas frecuentemente como hechos de cambio positivos importantes y se asocian más a la felicidad que a actividades laborales, académicas o económicas (Bilbao, Páez, Da Costa, \& Martínez-Zelaya, 2013).

La aplicación de los instrumentos requirió obtener autorización de los decanos de una universidad para utilizar los correos institucionales de los estudiantes y así fueron invitados 3000 de ellos a responder voluntariamente los instrumentos en la plataforma GoogleDocs. Se envío un recordatorio o segunda invitación a aquellos que al finalizar la segunda semana aún no habían completado los instrumentos. Se obtuvieron así 433 estudiantes (14.4\% del total) que completaron los instrumentos solicitados.

Se verificaron los supuestos de normalidad y homocedasticidad de las medidas consideradas en el análisis y la presencia o ausencia de diferencias en

1 Pololear o andar: chilenismos, el primero de los cuales significa 'mantener una relación sentimental con otro(a) con cierto grado de compromiso o formalidad', en tanto que el segundo significa 'una de menor compromiso o menor formalidad'. la distribución de las escalas usadas (EFS y CCPF), controlándose personalidad auto-adscrita, sexo y religión. Se efectuó un ANOVA factorial de 2X2X2 para identificar la presencia de interacciones entre las variables. Se realizaron comparaciones de medias mediante $t$ para muestras independientes y se utilizaron regresiones jerárquicas para identificar la configuración de conductas y estrategias que mejor predijesen la felicidad, describiendo las relaciones entre la EFS y la CCPF.

\section{Resultados}

\section{Felicidad de los universitarios según sexo, religión y personalidad}

En términos generales se observa que los estudiantes presentan niveles moderados de felicidad $(\mathrm{M}=$ 5.14 sobre 7; $D E=1.07$ ), sin diferencias significativas según sexo $\left(F_{[431]}=0.11 ; p=0.73\right)$, religión $\left(F_{[431]=34 ; p}=0.55\right)$ o personalidad auto-adscrita $\left(F_{[431]}=0.42 ; p=0.43\right)$.

Las mujeres realizan más conductas para procurarse felicidad que los hombres $\left(\mathrm{M}_{\text {mujeres }}=2.52 ; \mathrm{DE}\right.$ $\left.=0.23 ; M_{\text {hombres }}=2.47 ; \mathrm{DE}=0.25\right)$, siendo estas diferencias estadísticamente significativas $\left(F_{[432]}\right.$ $=3.83 ; p=0.05)$. Sin embargo, no se observan diferencias respecto a la eficacia de las conductas realizadas en procurarles felicidad $\left(F_{[432]}=2.87 ; p\right.$ $=0.09$ ). Así, aun cuando las mujeres realizan más actividades, estas no son más eficaces que las realizadas por los hombres. Ninguna de las estrategias realizadas por los hombres resulta ser significativamente superior a las de las mujeres. Sin embargo, se observan diferencias significativas en favor de los hombres $\left(F_{[432]} \geq 4.95\right.$; ps $\left.\leq 0.02\right)$ respecto a la frecuencia y eficacia de practicar ejercicio físico (ítems 17, a y b) como actividad para aumentar la felicidad. Los hombres realizan frecuentemente actividades deportivas o de ejercicio físico $(M=2.68$; $D E=0.85)$, mientras que la mujeres solo a veces $(\mathrm{M}=2.34 ; \mathrm{DE}=0.85)$. En cambio, las mujeres tienden a realizar significativamente más prácticas religiosas que los hombres $(M=2.11 ; D E=1.05$; $\left.F_{[432]}=10.86 ; p=0.001\right) y$ de mayor eficacia en provocarles felicidad que a los hombres $(\mathrm{M}=1.78$; 
$\left.D E=0.94 ; F_{[432]}=4.98 ; p=0.02\right)$. Las mujeres realizan significativamente más actividades propias del ocio pasivo $\left(\mathrm{M}_{\text {mujeres }}=2.56 ; \mathrm{DE}=0.28\right.$ y $\mathrm{M}_{\text {hombres }}$ $\left.=2.45 ; \mathrm{DE}=0.27, \mathrm{~F}_{[432]}=15.82\right)$ que los hombres y de mayor eficacia en proveerles felicidad $\left(M_{\text {muieres }}\right.$ $=2.96 ; \mathrm{DE}=0.39$ y $\mathrm{M}_{\text {hombres }}=2.83 ; \mathrm{DE}=0.42$; $\left.F_{[432]}=11.28 ; p=0.001\right)$. La misma tendencia se observa en el caso de las actividades inscritas en la estrategia de afiliación social, donde las mujeres las realizan más frecuentemente que los hombres $\left(\mathrm{M}_{\text {mujeres }}=2.95 ; \mathrm{DE}=0.43\right.$ y $\mathrm{M}_{\text {hombres }}=2.83 ; \mathrm{DE}$ $\left.=0.39 ; F_{[432]}=8.22 ; p=0.004\right)$, siendo también más eficaces para ellas $\left(\mathrm{M}_{\text {mujeres }}=3.26 ; \mathrm{DE}=0.4 \mathrm{y}\right.$ $\left.M_{\text {hombres }}=3.19 ; D E=0.4\right)$, aunque con diferencias marginalmente significativas $\left(F_{[432]}=3.67 ; p=\right.$ 0.05). Finalmente, los hombres realizan significativamente más actividades correspondientes a metas instrumentales que las mujeres $\left(\mathrm{M}_{\text {hombres }}=3.05\right.$; $\mathrm{DE}=0.62$ y $\left.\mathrm{M}_{\text {mujeres }}=2.91 ; \mathrm{DE}=0.63\right),\left(\mathrm{F}_{[432]}=\right.$ $5.26 ; p=0.02$ ), aunque la eficacia de dicha práctica no difiere significativamente entre ambos $\left(F_{[432]}=\right.$ $0.14 ; p=0.709)$.

La adscripción a una religión no tiene efectos significativos importantes sobre la mayoría de las conductas realizadas para aumentar la felicidad, ni tampoco sobre la eficacia de estas. Una sola excepción específica identificada aquí corresponde al comportamiento de rezar, que es significativamente más frecuente y también más eficaz en quienes se adscriben a una religión que en quienes no lo hacen $(M=2.37 ; D E=1.01$ vs $M=1.38 ; D E=0.7)$, respectivamente $(\mathrm{M}=2.98 ; \mathrm{DE}=0.94$ vs $\mathrm{M}=2.12$; $D E=1.14\left(F_{[432]} \geq 71.292\right.$; ps $\left.=0.001\right)$.

En lo que concierne a los rasgos de personalidad -extraversión ó introversión-y aun cuando no se observan diferencias en los niveles de felicidad, sí existen diferencias respecto a la frecuencia y eficacia de las conductas realizadas. Si bien, introvertidos y extrovertidos reportan como actividades más frecuentemente realizadas las inscritas en la estrategia "búsqueda de metas instrumentales", son los segundos quienes las realizan más frecuente y significativamente que los primeros $(M=3.03$ y $2.96 ; D E=0.59$ y 0.67 respectivamente) $\left(F_{[432]}=53.619 ; p=0.001\right)$. Respecto a la eficacia de las conductas realizadas se observa que introvertidos y extrovertidos, respectivamente, reportan como las conductas más efectivas aquellas correspondientes a las estrategias "intentos directos" $(\mathrm{M}=3.14$ y 3.39; $\mathrm{DE}=$ 0.54 y 0.51) y "afiliación social" ( $M=3.15$ y 3.30; $D E=0.41$ y 0.39). Para ambas estrategias hay diferencias estadísticamente significativas entre ellos ( $F s \geq 14.99 ;$ ps $=0.001$ ), siendo los extrovertidos quienes puntúan más alto en ambas. La estrategia menos frecuentemente utilizada por introvertidos y extrovertidos coincidió y fue "fiestas y discotecas" $(M=1.74$ y $2.09 ; D E=0.41$ y 0.49$)$ y también fue menos efectiva en sus efectos $(\mathrm{M}=2.52$ y $2.70 ; D E=0.57$ y 0.59 ). No obstante, existen diferencias significativas tanto para la frecuencia como para la eficacia de esta estrategia $\left(\mathrm{Fs}_{[432]}=\right.$ 10.59; ps = 0.001), de modo que los introvertidos la reportan como menos frecuente y menos eficaz.

La frecuencia y la eficacia de las conductas realizadas para procurarse felicidad predicen de forma significativa y positiva las puntuaciones de felicidad, aun cuando el porcentaje de varianza explicada es bajo $\left(R^{2}=0.19 ; F=52.23 ; p=0.001\right)$. Así, realizar frecuentemente conductas para aumentar la felicidad resulta eficaz, y se observa que la frecuencia de realización de las actividades pro felicidad es un mejor factor predictor de esta que la eficacia (Tabla 1).

\section{Análisis de quienes son más felices}

Los puntajes más altos de felicidad, cuartil cuatro de puntuaciones de felicidad, de 6 a 7 puntos, corresponden principalmente a quienes se autoadscriben como extrovertidos $\left(68.81 \% ; X^{2}[1]=\right.$ $20.945 ; p=0.001)$. Para este subgrupo $(n=110)$ las actividades más frecuentemente realizadas corresponden a búsqueda de metas instrumentales (M $=3.2 ; \mathrm{DE}=0.56)$, afiliación social $(\mathrm{M}=3.13 ; \mathrm{DE}$ $=0.37)$ y religiosas $(\mathrm{M}=2.05 ; \mathrm{DE}=1.09)$ (Figura 1), las que a su vez difieren significativamente entre sí $\left(t s_{[109]} \geq 19.75 ; p s=0.001\right)$. Respecto a la eficacia de cada una y difiriendo también significativamente entre sí $\left(t s_{[109]} \geq 25.38\right.$; ps $\left.=0.001\right)$, su orden cambia, ya que la más eficaz resulta ser la religiosa $(M$ $=2.85 ; D E=1.17)$, seguida por afiliación social 
TABLA 1

Coeficientes de predicción según dimensión Frecuencia y Efectividad de las actividades realizadas para aumentar la felicidad

\begin{tabular}{|c|c|c|c|c|c|}
\hline \multirow{2}{*}{ Modelo } & \multicolumn{2}{|c|}{ Coeficientes no estandarizados } & \multirow{2}{*}{$\begin{array}{c}\text { Coeficientes tipificados } \\
\text { Beta }\end{array}$} & \multirow[b]{2}{*}{$\mathrm{t}$} & \multirow{2}{*}{ Sig. } \\
\hline & $\mathrm{B}$ & Error típ. & & & \\
\hline (Constante) & 0.091 & 0.49 & & 0.18 & 0.85 \\
\hline $\begin{array}{l}\text { Uso general de conductas } \\
\text { para mejorar la felicidad }\end{array}$ & 1.38 & 0.22 & 0.31 & 6.08 & 0.00 \\
\hline $\begin{array}{l}\text { Eficacia general de conductas } \\
\text { para mejorar la felicidad }\end{array}$ & 0.54 & 0.15 & 0.18 & 3.46 & 0.001 \\
\hline
\end{tabular}

a. Variable dependiente: Escala de Felicidad

$(n=433)$

Fuente: elaboración propia

$(\mathrm{M}=3.40 ; \mathrm{DE}=0.42)$ y, finalmente, la búsqueda de metas instrumentales $(M=3.36 ; D E=0.53)$.

\section{Discusión y conclusiones}

Los estudiantes universitarios tienen un nivel moderado de felicidad, en convergencia con investigaciones previas para ese tipo de población (Argyle \& Lu, 1990; Moyano Díaz y Ramos, 2007), aunque sin diferencias según sexo ni personalidad autoadscrita. Las mujeres aparecen significativamente más activas que los hombres, desarrollando más conductas pro felicidad que ellos, muy particularmente, aquellas propias de las actividades religiosas, de ocio pasivo y afiliativas. Los hombres en cambio realizan más actividades físico-deportivas y orientadas a metas instrumentales, y ello es particularmente significativo en quienes se autoclasifican como extrovertidos y que son, a su vez, quienes obtienen mayor eficacia en lo que corresponde a la dimensión intentos directos. La dimensión fiestas y discotecas fue la menos practicada (junto con intentos directos) y la menos eficaz en procurarles felicidad. Aparentemente, la participación en

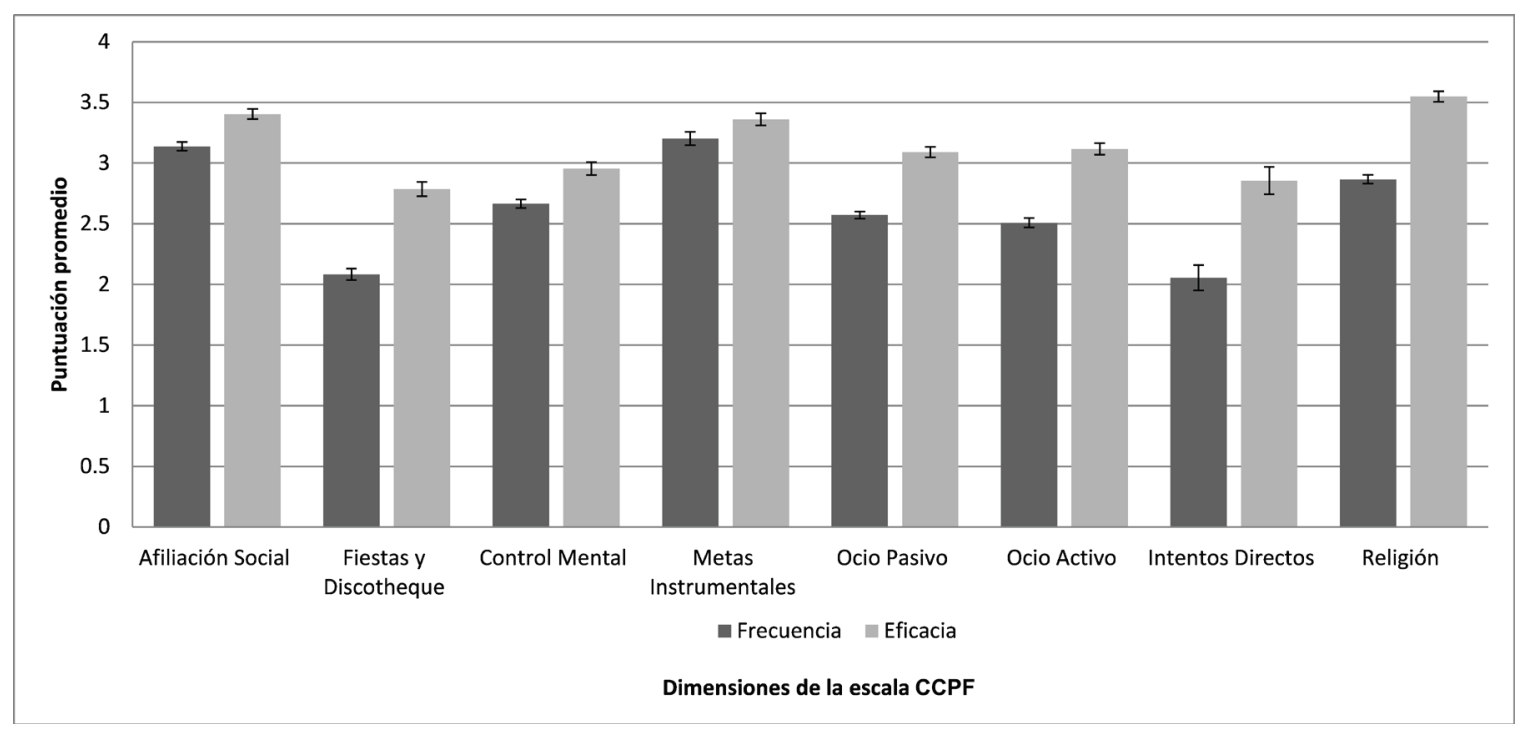

Figura 1. Comparación de promedios de frecuencia de uso y efectividad de las conductas realizadas para aumentar la felicidad del cuartil de jóvenes más felices $(n=110)$.

Fuente: elaboración propia 
esos eventos más bien incrementa la probabilidad y facilita que se produzcan otras conductas que sí son relevantes para aumentar la felicidad, como las conductas e intentos directos por ser feliz, los que sí aparecen como eficaces (conocer gente, conversar, compartir, sonreír).

Se observa que las mujeres ocupan su ocio principalmente en comportamientos de tipo pasivo y conductas sedentarias (Lemp \& Behn, 2008; Soto y Moyano Díaz, 2010) y ha sido sugerido que cuando ellas experimentan estados emocionales negativos son más proclives que los hombres a ingerir alimentos para mejorarlos (Grunberg \& Straub 1992). También ellas son más propensas a buscar interacción social cuando están deprimidas, lo que las hace practicar más la afiliación social, como por ejemplo, conversar sus problemas con amigas, la familia u otros. Así, la diferencia observada aquí entre hombres y mujeres respecto a la realización de actividades físicas ha sido convergente con lo reportado en otras investigaciones (por ejemplo, Melin et al., 2003).

Una de las conductas más eficaces para procurarse felicidad en los estudiantes es la búsqueda de metas instrumentales, tales como mejorar el rendimiento y alcanzar objetivos académicos. También se esperaba que quienes aparecieran más felices presentaran una mayor frecuencia de actividades destinadas a interactuar con otras personas, lo que efectivamente se observó en cuanto a que un $24 \%$ de los estudiantes más felices practicaban la interacción social, sin diferencias significativas según sexo.

Se encontró también apoyo a los resultados reportados en la literatura que cuestiona la universalidad de una relación positiva entre práctica religiosa y felicidad (Kesebir \& Diener, 2009) ya que no se encontró globalmente relación significativa entre ambas variables, pero sí en cambio, esa relación para el subgrupo más feliz de la muestra. El tipo de personalidad autorreportado (extravertido/introvertido) no genera diferencias en felicidad ni en la frecuencia de actividades propias de las estrategias control mental $(p=0.67)$ ni búsqueda de metas instrumentales ( $p=0.287$ ), ni en su efectividad ( $p$ $=0.111)$; tampoco en frecuencia y eficacia del ocio pasivo, el activo y la religión $(p \geq 0.06)$. Aunque ex- trovertidos e introvertidos no difieren en su nivel de felicidad, sí en cambio lo hacen en la eficacia de las estrategias para procurarse felicidad $(F=25.466$; $p=0.001$ ), donde los primeros realizan más actividades para sentirse felices que los segundos (afiliativas, por ejemplo, y de mayor eficacia), lo cual es respaldado por otras investigaciones (Tamir, 2009).

Igualmente ocurre con las actividades relativas a fiestas y a intentos directos (Tackh \& Lyumbrimsk, 2006). A su vez los introvertidos realizan una mayor cantidad de actividades de ocio pasivo y activo -y más eficaces- que los extrovertidos, lo que también es consistente con otros estudios (Tamir, 2009). Los más felices de esta muestra son mayoritariamente extrovertidos y realizan principalmente actividades referidas a metas instrumentales, afiliativas y religiosas, donde las más eficaces corresponden a este último tipo, seguidas por afiliativas y, finalmente, metas instrumentales. Dado que la frecuencia de realización de conductas pro felicidad ha resultado aquí un mejor predictor de esta que el factor eficacia, y entre aquellas la afiliación social y la búsqueda de metas instrumentales resultan más eficaces, parece aconsejable y saludable recomendar reunirse con otros a trabajar por metas o a practicar el ocio (o si se trata de alguien religioso, la religiosidad) y a celebrar o festejar, toda vez que ello sea posible.

\section{Referencias}

Alarcón, R. (2006). Desarrollo de una escala factorial para medir la felicidad. Revista Interamericana de Psicología, 40(1), 95-102.

Argyle, M., \& Lu, L. (1990). The happiness of extraverts. Personality and Individual Differences, 11(10), 1011-1017.

Bilbao, M. -A., Paez, D., Da Costa, S., \& Martinez, G. (2013). Extreme life-changing events, impact on basic beliefs and post-stress growth: Why positive events reinforce eudaimonic wellbeing more strongly than negative events undermine it. Terapia Psicológica, 31(1), 127-139.

Brandao, M. P., Pimentel, F. L., \& Cardoso, M. F. (2011). Impact of academic exposure on health status 
of university students. Revista de Saúde Pública, 45(1), 49-58.

Brunstein, J. C. (1993). Personal goals and subjective well-being: A longitudinal study. Journal of Personality and Social Psychology, 65(5), 1061-1070.

Casswell, S., Ru Quan You, \& Taisia Huckle (2011). Alcohol's harm to others: Reduced wellbeing and health status for those with heavy drinkers in their lives. Addiction, 106(6), 1087-1094.

Diener, E., \&. Seligman, M. (2002). Very happy people. Psychological Science, 13(1), 80-83.

Erisman, M., \& Roemer, L. (2010). A preliminary investigation of the effects of experimentally induced mindfulness on emotional responding to film clips. Emotions, 10(1), 72-82.

Gallup, G. (1998). Thankfulness: America's saving grace. Trabajo presentado en el National Day of Prayer Breakfast, Thanks-Giving Square, Dallas, TX.

Gratz, K. (2003). Risk factors and functions of deliberate self-harm: An empirical and conceptual review. Clinical Psychology: Science and Practice, 10(2), 192-205.

Grunberg, N. E., \& Straub, R. O. (1992). The role of gender and taste class in the effects of stress on eating. Health Psychology, 11(2), 97-100.

Gui, M., \& Stanca, L. (2009). Television viewing, satisfaction and happiness: Facts and fiction (Working Paper Series № 167). Milán, IT: University of MilanoBicocca, Dipartimento di Economia Politica.

Hsiao, E. T., \& Thayer, R. E. (1998). Exercising for mood regulation: The importance of experience. Personality and Individual Differences, 24(6), 829-836.

Huesmann, R., Moise-Titus, J., Podolski, C., \& Eron, L. (2003). Longitudinal relations between children's exposure to TV violence and their aggressive and violent behavior in young adulthood: 1977-1992. Developmental Psychology, 39(2), 201-221.

Kesebir, P., \& Diener, E. (2009). In pursuit of happiness: Empirical answers to philosophical questions. Social Indicators Research Series, 37, 59-74.

Langston, C. (1994). Capitalizing on and coping with daily-Life events-expressive responses to positive events. Journal of Personality and Social Psychology, 67(6), 1112-1125.
Larsen, J., \& McKibban, A. (2008). Is happiness having what you want, wanting what you have, or both? Psychological Science, 19(4), 371-377.

Laukka, P. (2007). Uses of music and psychological well-being among the elderly. Journal of Happiness Studies, 8(2), 215-241.

Lazarus, R., \& Lazarus, N. (1994). Passion and reason: Making sense of our emotions. New York: Oxford University Press.

Lemp, M., \& Behn, B. (2008). Utilización del tiempo libre de estudiantes del área de salud de la institución educacional Santo Tomás, Talca. Ciencia y Enfermería, 16(1), 53-62.

Lyubomirsky, S., \& Lepper, H. (1999). A measure of subjective happiness: Preliminary reliability and construct validation. Social Indicators Research, 46(2), 137-155.

Lyubomirsky, S., Sheldon, K. M., \& Schkade, D. (2005). Pursuing happiness: The architecture of sustainable change. Review of General Psychology, 9(2), 111-131.

McCullough, M., Emmons, R., \& Tsang, J. (2002). The grateful disposition: A conceptual and empirical topography. Journal of Personality and Social Psycho$\log y, 82(1), 112-127$.

Mcleod, J., Oakes, R., Copello, A., Crome, I., Egger, M., Hickman, M.,... Davey, G. (2004). Psychological and social sequelae of cannabis and other illicit drug use by young people: A systematic review of longitudinal, general population studies. The Lancet, 363(9421), 1579-1588.

Melin, R., Fugl-Meyer, K., \& Fugl-Meyer, A. (2003). Life satisfaction in 18-to 64-year-old Swedes: In relation to education, employment situation, health and physical activity. Journal of Rehabilitation Medicine, 35(2), 84-90.

Michalos, A. (2005). Arts and the quality of life: An exploratory study. Social Indicators Research, 71(1), $11-59$.

Moyano Díaz, E. y Ramos, N. (2007). Bienestar subjetivo:midiendo satisfacción vital, felicidad y salud en población chilena de la región del Maule. Universum, 22(2), 177-193.

Moyano, E., Flores, E. y Soromaa, H. (2009). Fiabilidad y validez de constructo del test MUNSH para medir 
felicidad en población de adultos mayores chilenos. Universitas Psychologica, 10(2), 567-580.

Newcomb, M., \& Bentler, P. (1988). Impact of adolescent drug use and social support on problems of young adults: A longitudinal study. Journal of Abnormal Psychology, 97(1), 64-75.

Orford, J., Krishnan, M., Balaam, M., Everitt, M., \& Van der Graaf, K. (2004). University student drinking: The role of motivational and social factors. DrugsEducation Prevention and Policy, 11(5), 407-421.

Páez, D., Mendiburo-Seguel, A. \& Martínez-Sánchez, F. (2013). Incremental validity of alexithymia, emotional coping and humor style on subjective and psychological well-being. Journal of Happiness Studies, 14(5), 1621-1637.

Pahl, K., Brook, J., Koppel, J., \& Yeon, J. (2011). Unexpected benefits: Pathways from smoking restrictions in the home to psychological well-being and distress among urban black and Puerto Rican Americans. Nicotine EF Tobacco Research, 13(8), 706-713.

Parylak, S., Koob, G., \& Zorrilla, E. (2011). The dark side of food addiction. Physiology E Behavior, 104(1), 149-156.

Pavez, P., Mena, L., \& Vera-Villarroel, P. (2012). El rol de la felicidad y el optimismo como factor protector de la ansiedad. Universitas Psychologica, 11(2), 369-380.
Pollner, M. (1989). Divine relations, social relations, and well-being. Journal of Health and Social Behavior, 30(1), 92-104.

Schimmack, U., Schupp, J., \& Wagner, G. (2008). The influence of environment and personality on the affective and cognitive component of subjective well-being. Social Indicators Research, 89(1), 41-60.

Schnittker, J. (2008). Happiness and success: Genes, families, and the psychological effects of socioeconomic position and social support. American Journal of Sociology, 114(Supl.), S233-S259.

Soto, E. y Moyano Díaz, E. (2010). Concepto y comportamiento de ocio y sus barreras en Maulinos, en el bicentenario de la República. Cap. 5 en: E. Moyano Díaz (Ed). Calidad de vida y psicología en el bicentenario de Chile. Santiago: Mármor.

Takch, C., \& Lyubomirsky, S. (2006). How do people pursue happiness? Relating personality happinessincreasing strategies, and well-being. Journal of Happiness Studies, 7(2), 183-225.

Tamir, M. (2009). Differential preferences for happiness: Extraversion and trait-consistent emotion regulation. Journal of Personality, 77(2), 447-470.

Teigen, K. (1997). Luck, envy, and gratitude: It could have been different. Scandinavian Journal of Psycho$\log y$, 38(4), 313-323.

Warner, R. M., \& Vroman, K. G. (2011). Happiness inducing behaviors in everyday life: An empirical assessment of "The How of Happiness". Journal of Happiness Studies, 12(6), 1063-1082. 